\title{
Setting Upper Limits on the Local Burst Rate Density of Primordial Black Holes Using HAWC
}

\author{
Kristi Engel and Alison Peisker \\ E-mail: klengel@umd.edu, peiskera@msu.edu \\ For the HAWC Collaboration ${ }^{\dagger}$ \\ For a complete author list and acknowledgements see PoS(ICRC2019)1177, \\ $\dagger$ https://www.hawc-observatory.org/collaboration/icrc2019.php
}

\begin{abstract}
Primordial Black Holes (PBHs) are black holes that may have been created by density fluctuations in the early Universe and could be as massive as supermassive black holes or as small as the Planck scale. It is believed that a black hole has a temperature inversely proportional to its mass and will thermally emit all species of fundamental particles via Hawking Radiation. PBHs with initial masses of $\sim 5 \times 10^{14} \mathrm{~g}$ (approximately one gigaton) should be expiring today with bursts of high-energy gamma radiation in the GeV-TeV energy range. The High Altitude Water Cherenkov (HAWC) Observatory is sensitive to particles with energies of $300 \mathrm{GeV}-100 \mathrm{TeV}$, which corresponds to the high end of the PBH burst spectrum. With its large instantaneous field of view of $\sim 2 \mathrm{sr}$ and a duty cycle above $95 \%$, the HAWC Observatory is well suited to perform an all-sky search for PBH bursts. We conducted a search on 959 days of gamma-ray data from HAWC by optimizing a previous gamma-ray burst transient search to the PBH burst energy spectrum, and placed the strongest upper limits on the local PBH burst rate density at the $99 \%$ confidence level.
\end{abstract}

Corresponding authors: Kristi Engel ${ }^{* 1}$, Alison Peisker ${ }^{2}$, Pat Harding ${ }^{2,3}$, Joshua Wood ${ }^{4}$, Israel Martinez-Castellanos ${ }^{1}$, Andrea Albert ${ }^{3}$, Kirsten Tollefson ${ }^{2}$

${ }^{1}$ University of Maryland, College Park

${ }^{2}$ Michigan State University

${ }^{3}$ Los Alamos National Laboratory

${ }^{4}$ University of Wisconsin, Madison

36th International Cosmic Ray Conference -ICRC2019-

July 24th - August 1st, 2019

Madison, WI, U.S.A.

${ }^{*}$ Speaker. 


\section{Introduction}

While there are no known processes in the current Universe than can create black holes with masses less than $\sim 1 M_{\odot}$, conditions in the early Universe were conducive to the formation of black holes with a wide range of masses [1]. These black holes, with masses ranging from the Planck mass to supermassive black holes, are called Primordial Black Holes (PBHs). PBH production in the early Universe would have broad observable consequences spanning from the largest scales (e.g., influencing the development of large-scale structure in the Universe), to the smallest scales (e.g., enhancing local dark matter clustering). In the present Universe, PBHs in certain mass ranges may constitute a fraction of dark matter [1,2].

Stephen Hawking convolved quantum field theory, thermodynamics, and general relativity to show that a black hole will thermally radiate ('evaporate') with a temperature inversely-proportional to its mass [3]. The emitted radiation consists of all fundamental particles with masses less than approximately the black hole temperature [4]. For those black holes of $\sim$ stellar mass and above, Hawking's radiation is nearly negligible. However, for small enough $\mathrm{PBHs}$, this process becomes the dominant governance of their evolution over time [5]. PBHs with initial masses of $\sim 5 \times 10^{14} \mathrm{~g}$ (approximately one gigaton) should be expiring today with bursts of high-energy gamma radiation in the $\mathrm{GeV}-\mathrm{TeV}$ energy range [6].

Confirmed detection of a PBH burst (evaporation event) would - beyond proving their existence and allowing the determination of their rate-density of evaporation and relic density-provide valuable insights into many areas of physics, including processes in the very early Universe and particle physics at energies higher than currently achievable by terrestrial accelerators. Even the non-detection of PBH burst events in dedicated searches would yield important constraints concerning the early Universe.

\section{The HAWC Observatory}

The High Altitude Water Cherenkov (HAWC) Observatory, a successor to the Milagro Observatory [7], is a very-high-energy (VHE) ground-based air shower array located on the side of the Sierra Negra volcano in Mexico at an altitude of 4,100 m above sea level. It has a wide field-of-view of $\sim 2 \mathrm{sr}$ and an operational energy range of $300 \mathrm{GeV}-100 \mathrm{TeV}$. HAWC consists of 300 water tanks in the main array encompassing a surface area of $22,000 \mathrm{~m}^{2}$. Each main tank is $7.3 \mathrm{~m}$ in diameter and $4.5 \mathrm{~m}$ deep, equipped with four (three $8^{\prime \prime}$ and one $10^{\prime \prime}$ ) upward-facing photomultiplier tubes (PMTs) anchored to the bottom of the tank. HAWC has completed installing and integrating an additional "outrigger" array comprised of 345 cylindrical tanks arranged in a concentric, circular, symmetric way around the main array, covering an additional instrumented area of $\sim 4.5$ times that of the main array. Each of these outrigger tanks is $1.55 \mathrm{~m}$ in diameter and $1.65 \mathrm{~m}$ deep with a single $8^{\prime \prime}$ PMT anchored at the bottom. However, the data used herein were taken before the inclusion of this new outrigger array.

In both the main array and the outriggers, the PMTs detect Cherenkov light from secondary particles created in extensive air showers induced by VHE gamma rays incident upon Earth's atmosphere. The main data acquisition system measures the arrival direction and energy of the VHE gamma rays by timing the arrival of the secondary particles on the ground and measuring the am- 
plitude of the PMT signals. The direction of the primary particle may be resolvable with an error between 0.1 and 2.0 degrees, depending on its energy and location in the sky. With these features and a high duty cycle of greater than $95 \%$, HAWC is able, and ideally suited, to continuously monitor the Northern Hemisphere sky for high-energy emission from gamma-ray transients, such as PBHs.

HAWC's wide field-of-view, day and night, eliminates the statistical restrictions some other detectors may experience. Its advantageous features are particularly important for detecting burst transients with emission durations shorter than, e.g., the slewing times of imaging atmospheric Cherenkov telescopes (IACTs). While HAWC has not yet had a confirmed detection of a gammaray burst (GRB), a PBH burst would have a much harder spectrum, making its detection much more likely. Also, as all PBH bursts HAWC might see would be within the closest 0.5 parsec, we do not have to deal with the extragalactic background light (EBL) cutoff or any similar restrictions faced when performing a GRB search.

\section{Theory}

\subsection{Primordial Black Hole Burst Spectrum}

As the PBH radiates, it continually loses mass and its temperature increases to very high energies. The manner in which the PBH expires depends on physics at this energy extrema. The energy spectrum is determined by the chosen high-energy particle physics model. In this work, as in [8], we assume the Standard Evaporation Model (SEM) as our emission and particle physics model. In the SEM [4, 9] (based on the Standard Model of particle physics), a PBH should directly radiate those fundamental particles whose Compton wavelengths $\left(\lambda_{c}=\frac{h}{m c}\right)$ are comparable with the size of the black hole. When the black hole temperature exceeds the Quantum Chromodynamics (QCD) confinement scale ( 250-300 MeV), quarks and gluons should be radiated [4, 10]. On astrophysical timescales, the final jet products will be photons, neutrinos, electrons, positrons, protons, and anti-protons.

In the SEM model, the black hole temperature $(T)$ can be expressed in terms of the remaining lifetime $(\tau)$ of the black hole (that is, the time left until the black hole finishes evaporating) as follows $[9,11]$ :

$$
T \simeq\left[4.8 \times 10^{11}\left(\frac{1 \mathrm{sec}}{\tau}\right)\right]^{1 / 3} \mathrm{GeV}
$$

for temperatures well below its ultimate or Planck temperature $\left(1.417 \times 10^{32} \mathrm{~K}\right)$. The emission rate increases as the black hole shrinks [10]. For black holes with temperatures greater than several $\mathrm{GeV}$ at the start of the observation, the time-integrated photon flux can be parameterized as [11]

$$
\frac{d N_{\gamma}}{d E_{\gamma}} \approx 9 \times 10^{35}\left\{\begin{array}{c}
\left(\frac{1 \mathrm{GeV}}{T}\right)^{3 / 2}\left(\frac{1 \mathrm{GeV}}{E_{\gamma}}\right)^{3 / 2} \mathrm{GeV}^{-1} \text { for } E_{\gamma}<T \\
\left(\frac{1 \mathrm{GeV}}{E_{\gamma}}\right)^{3} \mathrm{GeV}^{-1} \text { for } E_{\gamma} \geqslant T
\end{array}\right.
$$

for gamma ray energies $\mathrm{E} \gtrsim 10 \mathrm{GeV}$. This parameterization includes both directly radiated photons and those produced by the decay of other directly radiated species. Figure 1 shows the total gammaray spectrum for various $\mathrm{PBH}$ remaining lifetimes $(\tau)$ ranging from $0.01 \mathrm{~s}-100 \mathrm{~s}$. In this work, we examine remaining lifetimes, $\tau$, of $0.2 \mathrm{~s}, 1 \mathrm{~s}$, and $10 \mathrm{~s}$. 


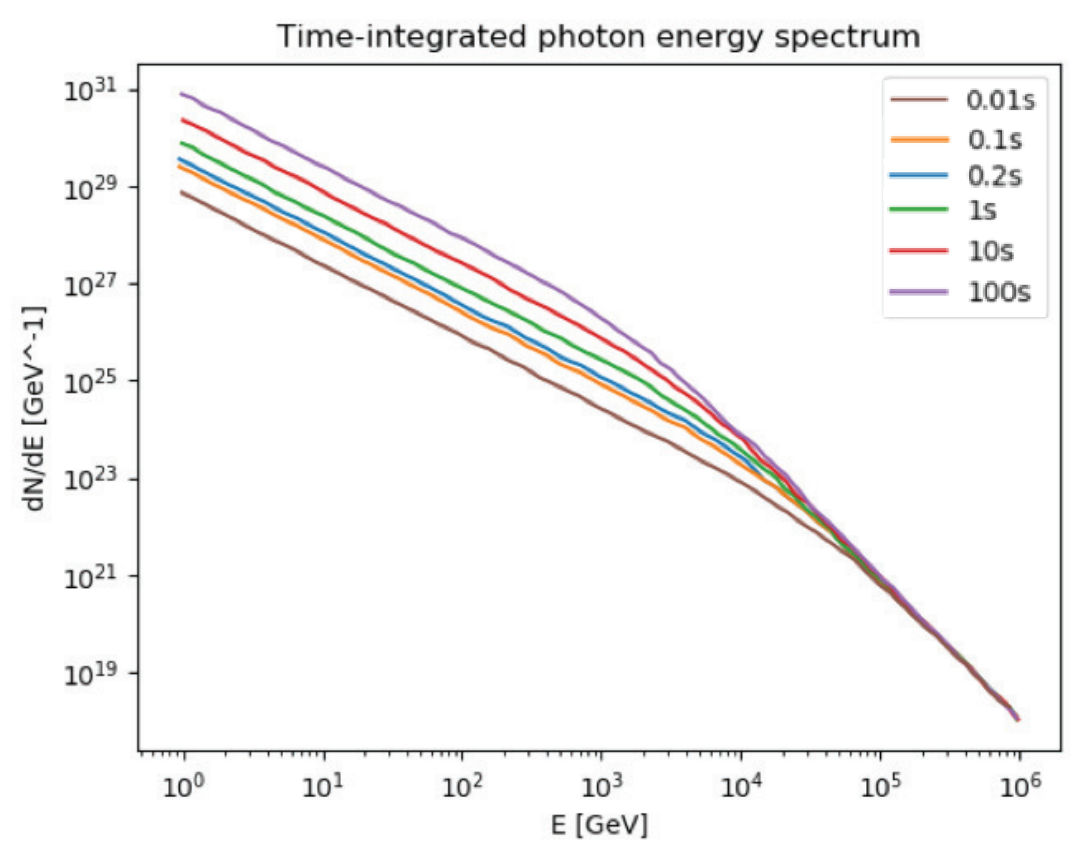

Figure 1: The photon spectrum, integrated over the final black hole evaporation lifetime intervals $\tau=0.01$, $0.1,0.2,1,10$, and 100 seconds. Adapted from [12].

\subsection{Creating a Model}

The first step in modeling PBH bursts is to simulate burst source points in HAWC's field-ofview. Assuming a local burst rate density of PBHs, $R$, of $10^{4} \mathrm{pc}^{-3} \mathrm{yr}^{-1}$, we used a Monte Carlo to randomly generate points out to 0.5 parsec uniformly in a $50^{\circ}$ cone about HAWC. We simulated PBHs with this burst rate because it is close to HAWC's predicted sensitivity [8], but it could have been chosen to be any positive non-zero value.

The parameterization of the time-integrated photon flux given in eq. (3.2) can be used to calculate the expected number of photons detectable by an observatory on the Earth's surface. For a PBH burst of duration $\tau$ at a non-cosmological distance $r$ and zenith angle $\theta$, the number of expected photons is

$$
\mu(r, \theta, \tau)=\frac{(1-f)}{4 \pi r^{2}} \int_{E_{1}}^{E_{2}} \frac{d N(\tau)}{d E} A(E, \theta) d E
$$

where $\mathrm{dN} / \mathrm{dE}$ is the $\mathrm{PBH}$ gamma ray spectrum integrated from remaining lifetime $\tau$ to 0 . The energies $E_{1}$ and $E_{2}$ correspond to the energy range of the detector, $A(E, \theta)$, is the effective area of the detector as a function of photon energy and zenith angle, and $f$ is the dead time fraction of the detector. Software was used to internally convolve HAWC's effective area with the assumed PBH spectrum and determine the expected signal from each simulated PBH in HAWC's field-of-view for each of the three remaining lifetimes to search.

Using this signal, we can calculate the probability (p-value) of obtaining $N$ counts given the background, $B$, for each event $i$, where $N=(\mu+B)$, using 


$$
\operatorname{prob}(\geqslant N)=\sum_{i=N}^{\infty} \frac{B^{i} e^{-B}}{i !}=1-\frac{\Gamma(N, B)}{\Gamma(N)} .
$$

These p-values are compiled into a histogram, $H_{p b h}$ for each duration to search $-0.2,1$, and $10 \mathrm{~s}$.

\section{Analysis}

\subsection{HAWC Transient Search Data}

The HAWC GRB program was established from the desire to leverage the full capacity of the HAWC Observatory's wide-field continuous monitoring of the TeV sky to discover GRB transients that occur at any time within the HAWC field-of-view, not just during the $\sim 50 \%$ of the time when satellites capable of providing GRB triggers are overhead [13]. It consists of two dedicated analyses: a self-triggered all-sky search, and a rapid response follow-up of GRBs reported by satellites [14]. Both methods are performed in real time and repeated on archival data when improved calibrations and reconstruction algorithms become available. It is the data from this first method that was optimized for the PBH burst spectrum and used to generate our model (described below).

The self-triggered all-sky analysis method continuously searches for GRB transients at energies $>100 \mathrm{GeV}$ with four sliding time windows of lengths $0.2,1,10$, and 100 seconds - corresponding to typical timescales of peak structures within GRBs. The search is performed by shifting each window forward in time and binning air shower events during that window using a grid of $2.1^{\circ} \times 2.1^{\circ}$ locally-smoothed square spatial bins covering all points within $50^{\circ}$ of detector zenith. Points outside a zenith angle of $50^{\circ}$ are excluded from the spatial search as most photons at the energies expected from a GRB signal originating from that relative angle do not have sufficient energy to reach HAWC. The fixed-width time windows, as well as the square bins, were chosen rather than attempting to fit a light curve profile in order to maintain the computation efficiency necessary for a full search of the HAWC field-of-view for all times [13]. The number of showers in each spatial bin is then compared to the expectation from charged cosmic ray backgrounds. From this, locations with an air shower excess corresponding to a post-trials false alarm rate of one event per day are considered candidates for GRB transients and are reported internally within HAWC. Thus far, there have been no significant detections of GRB transients since the inauguration of the full HAWC detector on March 19, 2015.

Beyond the application of the PBH spectrum described in Section 3.1, this transient search data was optimized for our analysis by adjusting the spatial and time window shifts to improve sensitivity. Additionally, the reporting threshold for events was lowered to more accurately reflect the kind of signal we would anticipate in HAWC from a PBH burst.

\subsection{Calculating an Upper Limit}

We began the analysis by choosing an approximate value for the local burst rate density of PBHs, $R$, of $\sim 10^{4} \mathrm{pc}^{-3} \mathrm{yr}^{-1}$ (the rate thrown in our Monte Carlo of simulated PBHs; Section 3.2). Using Poisson log likelihood calculations to determine the test statistic associated with $R$, we iterated this analysis over values of $R$ until we found the most probable value given the data and the 99\% limit on $R$; that is, the value of $R$ for which $T S=T S_{99}$. 
To determine the $99 \%$ limit on $R$, we began by calculating the test statistic for $R$ from Wilks' theorem [15],

$$
T S=2\left[\ln \left(\mathscr{L}_{1}\right)-\ln \left(\mathscr{L}_{0}\right)\right]
$$

where $\mathscr{L}_{0}$ is the background Poisson log-likelihood and $\mathscr{L}_{1}$ is our model Poisson log-likelihood. In general, the log-likelihood of a Poissonian with mean $\lambda$ for an IID sample of size $\mathrm{n}$ is given by

$$
\ln \left[\mathscr{L}\left(\lambda \mid x_{1}, x_{2}, \ldots x_{n}\right)\right]=-n \lambda+\left(\sum_{i=1}^{n} x_{i}\right) \ln (\lambda)-\ln \left(\prod_{i=1}^{n} x_{i} !\right)
$$

such that we can write, summing over bins $p$ instead of sample size,

$$
\ln \left(\mathscr{L}_{0}\right)=\sum_{p}\left[H_{\text {data }}(p) \ln \left(H_{b k g}^{\prime}(p)\right)-H_{b k g}^{\prime}(p)\right]
$$

where $H_{\text {data }}$ is a histogram of the HAWC data p-values produced by HAWC's GRB program $[14,13]$ and $H_{b k g}^{\prime}$ is a histogram of p-values generated by Monte Carlo using HAWC background distributions, also from the GRB analysis, scaled by the time of the searched data, and

$$
\ln \left(\mathscr{L}_{1}\right)=\sum_{p}\left[H_{\text {data }}(p) \ln \left(H_{\text {model }}(p)\right)-H_{\text {model }}(p)\right]
$$

where $H_{\text {model }}(p)=H_{b k g}^{\prime}(p)+H_{p b h}^{\prime}(p) ; H_{p b h}^{\prime}$ being scaled to both the rate of PBHs used to calculate $H_{p b h}$ and the time of the "searched" Monte Carlo. Note that both expressions neglect the factorial term in the likelihood eq. (4.2) as it will cancel when evaluating eq. (4.1).

We then found the value of $R$ that yielded the largest TS value from eq. (4.1), $T S_{\max }$. From this, iterating over $R$, the burst rate that satisfies the TS value corresponding to a $99 \%$ confidence level is the upper limit:

$$
T S_{99}=T S_{\max }-5.41
$$

where 5.41 is the 1-sided confidence limit value corresponding to $99 \%$.

The limit, corresponding to a remaining lifetime $(\tau)$ of $0.2 \mathrm{~s}$, is

$$
\dot{\rho}<3296 \mathrm{pc}^{-3} \mathrm{yr}^{-1}
$$

the strictest limit yet placed on the local PBH burst rate density.

\section{Discussion}

The final 99\% confidence level upper limits on the PBH burst rate density are shown in Figure 2. The best of these results, corresponding to a remaining lifetime of $0.2 \mathrm{~s}$, is also presented in Table 1 for direct comparison with the results of other direct searches for PBHs. We note that HAWC uncertainties - systematic or statistical—have not been included in this study. All limits shown in Figure 2 were obtained based on the PBH Standard Emission Model $[4,16]$. 


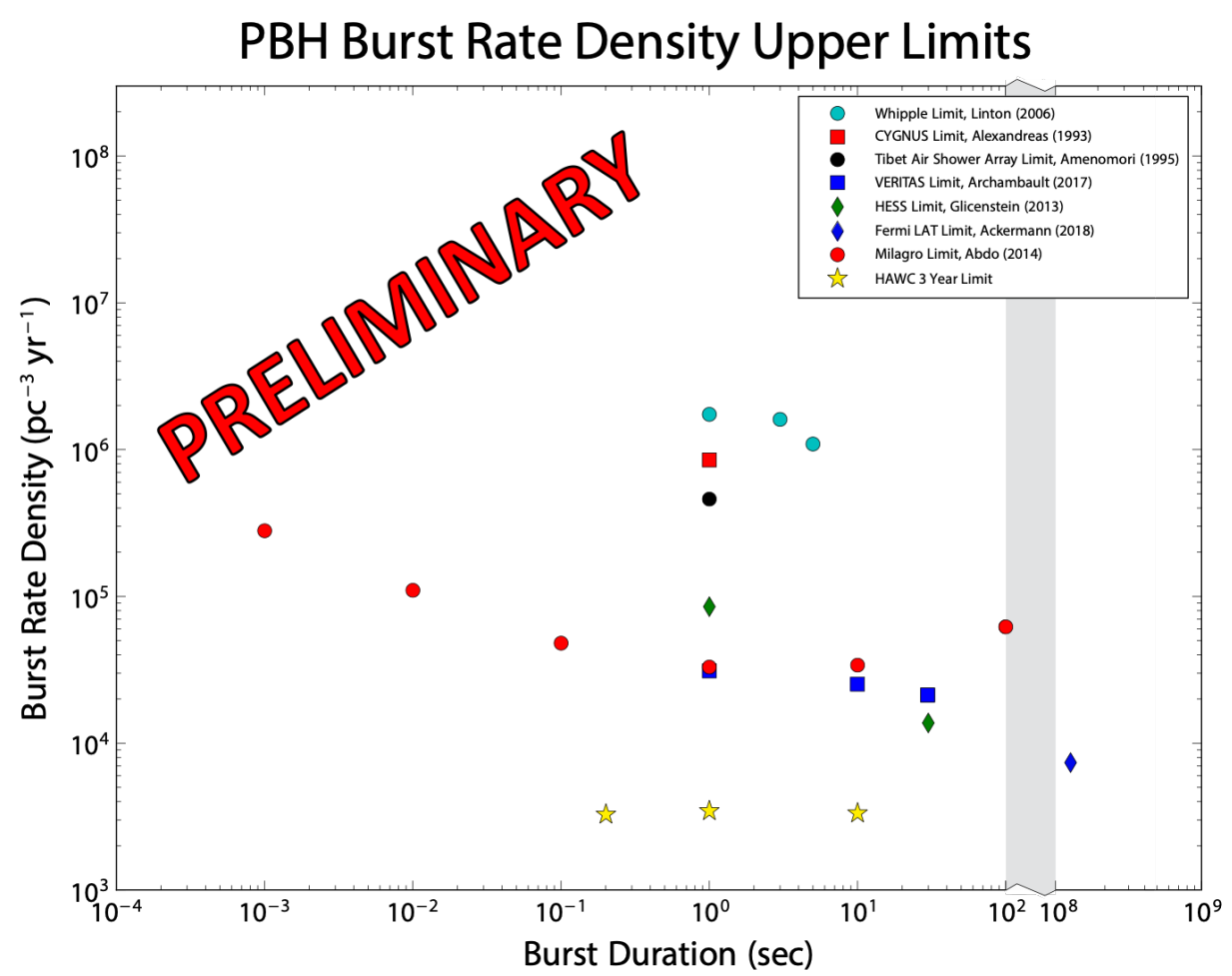

Figure 2: Comparison of the HAWC 99\% confidence level upper limits at 0.2, 1, and 10s with the limits from Whipple [17], CYGNUS [18], the Tibet Air Shower Array [19], VERITAS [20], H.E.S.S. [5], FermiLAT [21], and Milagro [8].

\begin{tabular}{c||c|c|c} 
Experiment & Burst Rate Upper Limit & Search Duration & Reference \\
\hline \hline Milagro & $36000 \mathrm{pc}^{-3} \mathrm{yr}^{-1}$ & $1 \mathrm{~s}$ & {$[8]$} \\
VERITAS & $22200 \mathrm{pc}^{-3} \mathrm{yr}^{-1}$ & $30 \mathrm{~s}$ & {$[20]$} \\
H.E.S.S. & $14000 \mathrm{pc}^{-3} \mathrm{yr}^{-1}$ & $30 \mathrm{~s}$ & {$[5]$} \\
Fermi-LAT & $7200 \mathrm{pc}^{-3} \mathrm{yr}^{-1}$ & $1.26 \times 10^{8} \mathrm{~s}$ & {$[21]$} \\
HAWC 3 yr. & $3300 \mathrm{pc}^{-3} \mathrm{yr}^{-1}$ & $0.2 \mathrm{~s}$ & This Work
\end{tabular}

Table 1: The strictest limit for each of the five detectors most sensitive to direct PBH studies.

Planned future work to improve this analysis includes the determination of uncertainties for these results, as well as a follow-up independent PBH study ideally working directly with HAWC's signal rather than probability values and incorporating data from the newly-added outrigger array. Statistical improvements for such a study would include calculation of likelihood in time in addition to that currently being done spatially, as well as stacking of these likelihoods to ensure the signal is well-defined for any zenith angle.

\section{References}

[1] B. J. Carr, K. Kohri, Y. Sendouda, and J. Yokoyama, Phys. Rev. D81 (2010) 104019. 
[2] B. Carr, F. Kuhnel, and M. Sandstad, Phys. Rev. D94 (2016) 083504.

[3] S. W. Hawking, Nature 248 (1974) 30 - 31.

[4] J. H. MacGibbon and B. R. Webber, Phys. Rev. D 41 (1990) 3052 - 3079.

[5] H.E.S.S. Collaboration, J.-F. Glicenstein, A. Barnacka, M. Vivier, and T. Herr, Limits on Primordial Black Hole evaporation with the H.E.S.S. array of Cherenkov telescopes, in Proceedings, 33rd International Cosmic Ray Conference (ICRC2013): Rio de Janeiro, Brazil, July 2-9, 2013, p. 0930, 2013. arXiv:1307.4898.

[6] J. H. MacGibbon, B. J. Carr, and D. N. Page, Phys. Rev. D 78 (2008) 064043.

[7] T. M. Collaboration and R. A. et al., NIM-A 449 (2000) 478 - 499.

[8] A. A. Abdo et al., Astropart. Phys. 64 (2015) 4-12.

[9] J. H. MacGibbon, Phys. Rev. D 44 (1991) 376 - 392.

[10] D. N. Page and S. W. Hawking, ApJ 206 (1976) 1 - 7.

[11] V. B. Petkov, E. V. Bugaev, P. A. Klimai, M. V. Andreev, V. I. Volchenko, G. V. Volchenko, A. N. Gaponenko, Z. S. Guliev, I. M. Dzaparova, D. V. Smirnov, A. V. Sergeev, A. B. Chernyaev, and A. F. Yanin, Astronomy Letters 34 (2008) 509 - 514.

[12] T. N. Ukwatta, D. R. Stump, J. T. Linnemann, J. H. MacGibbon, S. S. Marinelli, T. Yapici, and K. Tollefson, Astropart. Phys. 80 (2016) 90-114.

[13] J. R. Wood. PhD thesis, University of Maryland, College Park, 2016.

[14] HAWC Collaboration, J. Wood, PoS ( ICRC2017) 619 (2018).

[15] S. S. Wilks, The Annals of Mathematical Statistics 9 (1938) 60-62.

[16] F. Halzen, E. Zas, J. H. MacGibbon, and T. C. Weekes, Nature 353 (1991) 807-815.

[17] E. T. Linton and et al., Journal of Cosmology and Astroparticle Physics 01 (2006) 013.

[18] D. E. Alexandreas and et al., Physical Review Letters 71 (1993) 2524.

[19] Tibet Air Shower Array Collaboration, M. Amenomori et al., Search for 10 TeV Gamma Bursts from Evaporating Primordial Black Holes with the Tibet Air Shower Array, in Proceedings, 24th International Cosmic Ray Conference (ICRC1995): Rome, Italy, August 28 - September 8, 1995, p. 112.

[20] VERITAS Collaboration, S. Archambault, POS ( ICRC2017) 691 (2018).

[21] Fermi-LAT Collaboration, M. Ackermann et al., Astrophys. J. 857 (2018) 49. 